\title{
Energy Management Systems and tertiary regulation in hierarchical control architectures for islanded micro-grids
}

\author{
M. L. Di Silvestre, M. Graells, J. M. Guerrero, A. C. Luna, L. Mineo, N. Q. Nguyen, E. Riva Sanseverino, \\ and J. C. Vasquez
}

\begin{abstract}
In this paper, the structure of the highest level of a hierarchical control architecture for micro-grids is proposed. Such structure includes two sub-levels: the Energy Management System, EMS, and the tertiary regulation. The first devoted to energy resources allocation in each time slot based on marginal production costs, the latter aiming at finding the match between production and consumption satisfying the constraints set by the EMS level about the energy production in each time slot. Neglecting the efficiency of the different energy generation systems as well as that of the infrastructure for electrical energy distribution, the problem dealt with by the EMS sub-level is linear and can be solved by well known Linear Programming optimization procedures. The tertiary sub-level, below the EMS, optimizes mainly technical objectives and requires the solution of the Optimal Power Flow problem. After a review of the state of the art on the topic, the higher control sub-levels are described and an application is proposed. The application shows the efficiency of Mixed Integer Linear Programming methods for cost minimization of the energy production systems for microgrids. Also the formulation of the OPF problem is briefly outlined to describe the interaction between the two sub-levels
\end{abstract}

Index Terms-Energy management systems, micro-grids, optimization, linear programming.

\section{INTRODUCTION}

$\mathrm{T}$ HE problem of creating a control architecture for microgrids operation is a quite debated subject on the literature [1]. However the role of the higher levels in such architecture is not yet very well clear. Some papers argue that their role is mostly related to generation units scheduling for economic purposes and do not care about technical criteria [2-4], others only argue that the aim is primarily related to technical features such as power losses minimizations [5-6]

Copyright (c) 2011 IEEE. Personal use of this material is permitted. However, permission to use this material for any other purposes must be obtained from the IEEE by sending a request to pubs-permissions@iee.org.

Di Silvestre M. L., Riva Sanseverino E., Quang N. N. and Mineo L.are with the University of Palermo (corresponding author: 003909123860262; fax: 0039091488452; e-mail: eleonora.rivasanseverino@unipa.it).

Guerrero J.M. , Luna A.C., and Vasquez J. C. are with the Institute of Energy Technology, Aalborg University, 9220, Denmark.

Graelles M. is with Chemical Engineering Department, Universitat Politècnica de Catalunya (e-mail: moises.graells@upc.edu). synchronization issues and grid detection [7-9]. Such higher levels are either referred to as Energy Management Systems in the first case, to Tertiary regulation, in the second case.

Only a few cases in the literature are concerned with consider integrated architectures with the lower levels of the overall control system which account for technical issues such as power loss optimization and stability [10].

The aim of this paper is to propose a new structure of higher levels control, composed of two sub-levels: the energy manager and the optimal power flow optimizer. See figure 1 below. The first sublevel gives the lower sublevel the inputs for the optimal power flow solution.

To cope with the uncertainties related to the inherent unpredictability of renewable sources and loads, the algorithms supporting the sub-levels can be run with a rolling horizon approach, continuously updating predictions and thus schedulings.

\section{ENERGy MANAGEMENT PROBLEM Formulation}

It is well known that both in transmission and in distribution systems the basic problem of voltage and frequency regulation arises when generation cannot follow with the required speed and the loads variations.

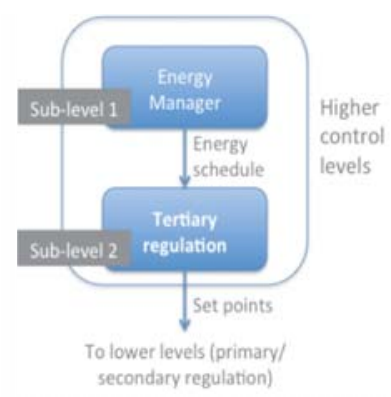

Fig. 1 Higher control levels for micro-grids.

In micro-grids [11], where generation and consumption are quite close and where Renewable Energy Sources, RES, is strongly present, the problem is very serious, especially in islanded condition. Moreover, it is a crucial issue in small systems how such load following activity is partitioned 
between the different generation units, also based on their ability to follow the loads and RES dynamics.

In some networks, in fact, the main power oscillations are caused by the renewable injections [4],[12]-[23]. In this case, the amount of energy provided for the regulating purposes, called regulating energy, should be evaluated according to the power fluctuations deriving from Renewable Energy Sources.

This regulating energy, assumed with negligible economic impact or at least much less than that for supplying the base load, cannot be scheduled according to economic criteria, but should be made available according to technical constraints; the latter being stricter when the system operates in islanded mode. Such value is also defined based on the elementary time interval chosen for the energy manager to solve the problem: the regulating energy is as smaller as the elementary time interval for scheduling is tight since there is less need for regulation, if scheduling of energy resources and match between production and consumption is almost real time.

Usually, the elementary time interval for scheduling is $15^{\prime}$ or one hour. In the present paper it is assumed a time slot $h=1 \mathrm{~h}$.

\section{A. Generation Unit classification}

In a micro-grid, such as in standard power transmission systems, a broad classification of generation units can be done referring to the dynamic of the generation units (also storage units can be classified accordingly, based on their response times and on their storage capacity) in [24], [25]:

1. Those providing the base load; these have slow ramping times, typically tens of minutes (Base units).

2. Those providing the regulation service to match generation and demand (fluctuations); these have fast ramping times, typically seconds (Peaking units).

3. Those providing both services; these have fast ramping times, typically seconds.

The first type of generators cannot be controlled every few minutes and typically work at rated power, supplying the base load of the scheduling horizon. Having slow response times, they can be scheduled hourly or every fifteen minutes.

The second type of generators will share the amount of power that can vary within the elementary time interval of ten to fifteen minutes. The control laws of the primary regulators in type 2 generators are of this type:

$$
\begin{aligned}
& P_{g}=-K_{g}\left(f-f_{0}\right) \\
& Q_{g}=-K_{g}\left(V-V_{0}\right)
\end{aligned}
$$

The third type of generators can both supply the base load and the regulating service contributing to regulation with a dynamic that depends on the generator's type and interface. The control laws of the primary regulators in type 3 generators are of this type:

$$
\begin{aligned}
& P_{g}=P_{g 0}-K_{g}\left(f-f_{0}\right) \\
& Q_{g}=Q_{g 0}-K_{g}\left(V-V_{0}\right)
\end{aligned}
$$

\section{B. Loads management}

Also loads can take part to regulation and can be managed according to economic criteria. They can be divided into two different classes:

- Manageable loads

- Unmanageable loads.

The first type of loads is essentially deferrable loads. For the sake of simplicity, the Energy Manager will consider a manageable load as an energy block, whose area represents the energy $\left(U_{\min }\right)$ required by its working cycle in 1 hour.

If load $\mathrm{Li}$ consists of a single shiftable appliance, the total energy required, $E t_{L i \_s}$, in $24 \mathrm{~h}$ is set as:

$E t_{L i_{-} s}=\sum_{h=1}^{24} E_{L i_{-} s h i f t}(h)=\sum_{h=1}^{24} \delta_{L i}(h) \cdot U_{\min }$

where $E_{L i \text { shift }}(h)$ is the energy supplied to the i-th load at hour h, $\delta_{L i}(h)$ is a binary variable taking the value 1 if the manageable load is working in h time slot, 0 otherwise.

If load $\mathrm{Li}$ is composed of a set of appliances of which only some are deferrable (partially manageable load), the energy required by the load at hour h can be described as:

$$
\begin{aligned}
E_{L i}(h) & =E_{L i_{-} \text {shift }}(h)+E_{L i_{-} \text {noshift }}(h)= \\
& =\sum_{k=1}^{K_{s h}} \delta_{L i_{k}}(h) \cdot U_{\text {min }_{k}}+E_{L i_{-} \text {noshift }}(h)
\end{aligned}
$$

In this case, the shiftable part $E_{L i_{-} \text {shift }}(h)$ is a multiple of the amount of energy $U_{\text {mink }}$ requested by each of the $K_{s h}$ appliances constituting the deferrable load. $\delta_{L i_{k}}(h)$ is a binary variable taking value 0 or 1 respectively if the k-th appliance is on or off at time h; $E_{L i_{-} \text {noshift }}(h)$ is the energy of the non-shiftable part of the load.

The total energy in $24 \mathrm{~h}$ is the maximum energy that is required to supply all the shiftable appliances of the considered load $L_{i}$. It can be set as:

$$
E t_{L i_{-} s}=\sum_{h=1}^{24} E_{L i_{-} s h i f t}(h)=\sum_{h=1}^{24}\left(\sum_{k=1}^{K_{s h}} \delta_{L i_{k}}(h) \cdot U_{\text {min }_{k}}\right)
$$

\section{Problem Formulation}

Let's assume that the regulation is carried out through VSC converters interfacing the generation units [1] and let's assume that all generation units of type 2 and 3 have this type of interface to the grid. In each time slot $h$, the EMS will decide:

- which deferrable load will be supplied

- $\quad$ what type 1 and 3 units are to be turned ON and what energy they will have to inject in the micro-grid

Let's assume that the studied micro-grid is islanded and includes $n_{G}$ generators and $n_{L}$ loads. Loads $1,2 \ldots n_{d}$ are deferrable loads (of which $n_{p}$ are partially manageable loads). $n_{G 1}$ generators are of type1, $n_{G 2}$ generators are of type 2 and $n_{G 3}$ generators are of type 3 , with: 
$n_{G}=n_{G 1}+n_{G 2}+n_{G 3}$

\section{Objective Function:}

The Energy Manager must solve the linear problem described by the following equation:

$$
\begin{aligned}
\text { Cost } & =\sum_{j=1}^{n_{G 1}} \sum_{h=1}^{24}\left(C_{G 1_{-} j} \cdot E_{G 1_{-} j}(h)\right)+\sum_{j=1}^{n_{G 2}} \sum_{h=1}^{24}\left(C_{G 2_{-} j} \cdot E_{G 2_{-} j}(h)\right)+ \\
& +\sum_{j=1}^{n_{G 3}} \sum_{h=1}^{24}\left(C_{G 3_{-} j} \cdot E_{G 3_{-} j}(h)\right)
\end{aligned}
$$

where: $C_{G 1}{ }_{j}, C_{G 2_{-} j}$ and $C_{G 3_{-} j}$ are the unitary cost of energy supplied by the j-th unit of type 1, type 2 and type 3 respectively; $E_{G 1_{-} j}(\mathrm{~h}), E_{G 2_{-} j}(\mathrm{~h})$, and $E_{G 3_{-} j}(\mathrm{~h})$ are the generated energy at each time $\mathrm{h}$ by the $\mathrm{j}$-th generator of type 1 , type 2 and type 3 respectively.

\section{Constraints}

In each time slot $h=1,2 \ldots 24$, the problem is subjected to the following constraints:

1) Energy balance between loads and generation. The energy supplied by generation units must equal the energy required by the loads.

$\sum_{j=1}^{n_{G 1}} E_{G 1_{-} j}(h)+\sum_{j=1}^{n_{G 2}} E_{G 2_{-} j}(h)+\sum_{j=1}^{n_{G 3}} E_{G 3_{-} j}(h)=\sum_{j=1}^{n_{L}} E_{L}(h)$

The total energy of the loads, $E_{L}(h)$, is composed by:

- the total shifted energy of the $n_{d}$ shiftable loads (included the shifted parts of the $p$ partial manageable loads of $\mathrm{K}_{\mathrm{sh}}$ kind);

- the total energy of the non-shiftable part of $n_{p}$ partially manageable loads;

- the total energy of $n_{L}-n_{d}$ non shiftable loads.

$$
E_{L}(h)=\sum_{i=1}^{n_{d}} \sum_{k=1}^{K_{s h}} \delta_{L i_{k}}(h) \cdot U_{\min _{k}}+\sum_{i=1}^{n_{p}} E_{L i_{-} n o s h i f t}(h)+\sum_{i=1}^{n_{L} n_{d}} E_{L i}(h)
$$

2) Constraint on power supplied by type 1 Generators (as gas micro-turbines generators), that have to range between $P_{\text {gmin }}$ and $P_{\text {gmax }}$ :

$P_{\text {gmin }} \cdot \Delta t \leq E_{g i}(h) \leq P_{\text {gmax }} \cdot \Delta t, \quad j=1,3$

where $\Delta t$ is the width of the elementary time interval.

3) Constraint on each storage unit:

- constraint on power charge and discharge $P_{\text {sto }}(h)$ from and to the battery:

$$
P_{\text {sto_min }} \leq P_{\text {sto }}(h) \leq P_{\text {sto__max }}(h)
$$

- constraint on the saved energy $E_{\text {sto }}(h)$ :

$$
E_{\text {sto_min }}(h) \leq E_{\text {sto }}(h) \leq E_{\text {sto_max }}(h)
$$

$E_{\text {sto_max }}(h)$ is the capacity of the storage unit; $E_{\text {sto_min }}(h)$ takes into account the fact that the battery must have enough energy available in the event of fluctuation to support the system if necessary, according to its dynamic characteristics (see subsection A, paragraph II)

- the energy saved in the battery is equal to the energy in the previous time ( $h-1)$ minus the energy that is being used:

$$
E_{\text {sto }}(h)=\left\{\begin{array}{lc}
\text { Charge o }-P_{\text {sto }}(h) \cdot \Delta t & h=1 \\
E_{\text {sto }}(h-1)-P_{\text {sto }}(h) \cdot \Delta t & 2 \leq h \leq 24
\end{array}\right.
$$

where Charge 0 is the initial condition of the storage unit.

-the charge at the last elementary unit of time has to be equal to the charge at the first elementary unit of time:

$$
\sum_{h=1}^{23} E_{\text {sto }}(h+1)-E_{\text {sto }}(h)=0
$$

The energy delivered in 24 hours by a storage system must equal zero. The constraint about the number of maneuvers is neglected because it is hypothesized that the battery is li-ion based technology.

\section{APPLICATION}

The test system, shown in figure 3, is a LV system not connected to the main grid and is supplied through three generation buses.

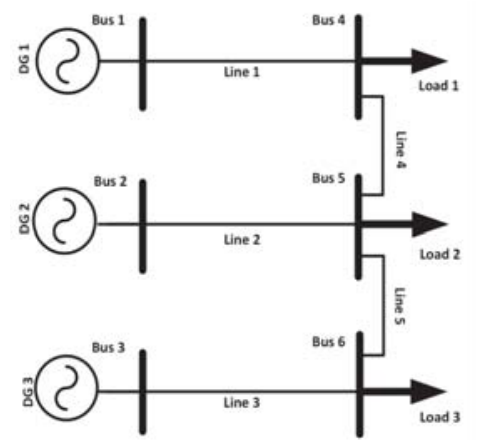

Fig. 3. 6 bus test system

The test system includes two type I generators, DG1 and DG3; they are microturbines with rated power comprised between 10 and $30 \mathrm{~kW}$. The ramping time is of 10-15 minutes. They can be scheduled every hour. The generator DG2 is a storage system. It has a ramping time of less than a minute, therefore it is a type 3 generator and has a capacity of $40 \mathrm{kWh}$. At the same bus there is a photovoltaic plant (PV plant) with peak power $40 \mathrm{kWp}$. Its Energy profile along $24 \mathrm{~h}$ is reported in figure 4.

Load 1 and Load 2 at buses 4 and 5 are residential loads partially manageable, with peak power of $22.5 \mathrm{~kW}$. Their load profile is shown in figure 5. The shiftable part in the time $h$ consists of three appliances of the same kind, with $U_{\text {min }}=0.75 \mathrm{kWh}$. Load 3 is not manageable. Thus, the inputs describing the loads are 5 sets of data: 
$E_{L i_{-} \text {shift }}(h), E_{L i_{-} \text {noshift }}(h), E_{L 3} \quad i=1,2$

For the shiftable loads, the total energy in $24 \mathrm{~h}(\mathrm{kWh})$ are set:

$E t_{L i_{-} s}=\sum_{h=1}^{24} E_{L i_{-} \text {shift }}(h) \quad i=1,2$

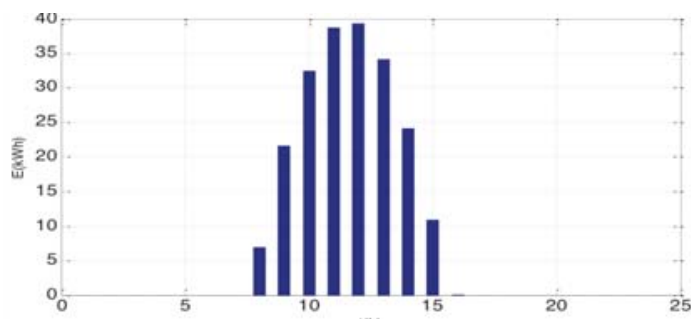

Fig. 4. Energy profile for the PV plant at bus 2 in the test system

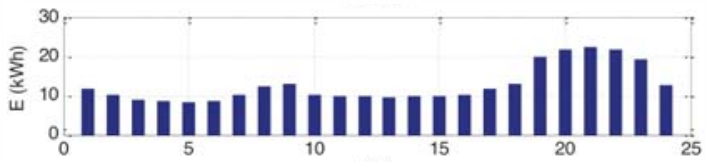

$\stackrel{t(h)}{\text { Load } 2}$

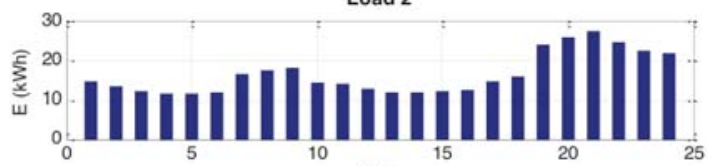

$t$ (h)

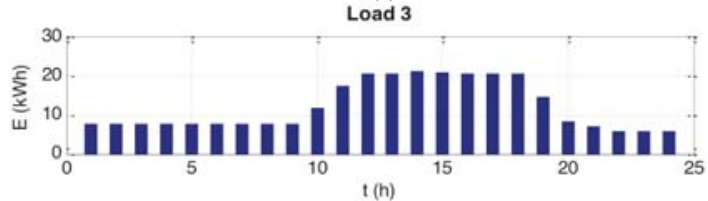

$\mathrm{t}(\mathrm{h})$

Fig. 5. Loads profile before optimization for the 6 bus test system

\section{A. Results and discussion}

The solver used is based on Mixed Integer Linear Programming and the model is implemented in GAMS. The operating cost obtained with the optimizer by using the parameters that are included in the Table I in 24 hour is 8.5037 $€$.

TABLE I

INPUT PARAMETERS

\begin{tabular}{cc}
\hline \hline \multicolumn{2}{c}{ Value } \\
\hline Parameter & 20 \\
\hline Charge $_{0}[\mathrm{kWh}]$ & 0.01 \\
$c_{1}[€ / \mathrm{kWh}]$ & 0.013 \\
$c_{3}[€ / \mathrm{kWh}]$ & 40 \\
$E_{\text {sto_max }}[\mathrm{kWh}]$ & 4 \\
$E_{\text {sto_min }}[\mathrm{kWh}]$ & 8 \\
$P_{\text {sto_max }}[\mathrm{kW}]$ & -4 \\
$P_{\text {sto_min }}[\mathrm{kW}]$ & \\
\hline \hline
\end{tabular}

The scheduling of the energy generated by the generation units are presented in the figure 7 . Generator 3 is used less time than the 1 because it has a slightly higher operating cost per unit. Generator 2 comprises a PV generator and a storage system.
As a matter of fact, the energy of generator DG2 (magenta) is indeed composed by the addition of the energy of the PV plus the energy of the storage, see figure 6 . The state of charge of the latter is shown in figure 7 . Between $h=8$ and $h=15$, part of the energy produced by the PV is used to charge the battery rather than to feed the grid.

The load is scheduled as shown in figure 8 .

The demand and generation are compared in figures 9 . The majority of demand is shifted to the time when generator 2 provides more energy.
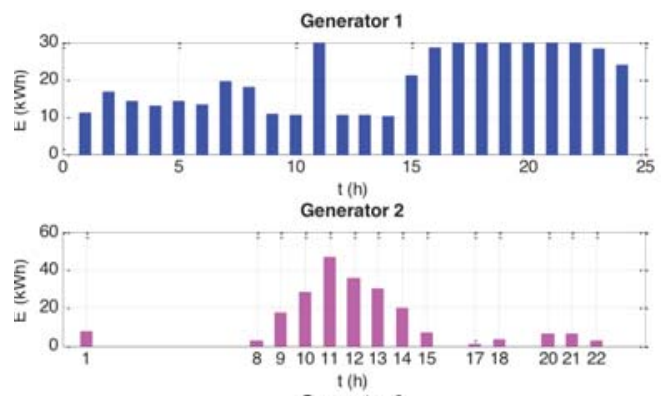
Generator 3

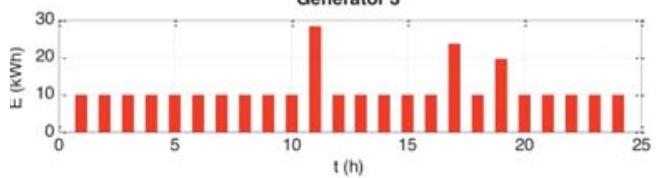

Fig. 6. Scheduling of the energy generated by the generation units

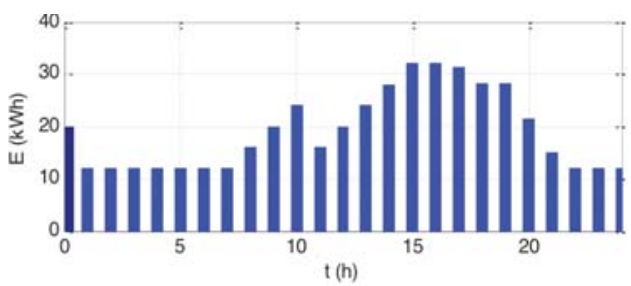

Fig. 7. State of charge of storage

Therefore, hour by hour, the OPF of tertiary regulation will get a new economically devised optimal operation set point for generator DG2 which is an inverter interfaced unit. The operating point will range around the economic set point defined through the EMS. Starting from the set point devised by the EMS, the OPF [5] adjusts the generation to follow the unexpected loads and generators fluctuations. The power set points of the generators are reported in table II below.

TABLE II

INPUT PARAMETERS

\begin{tabular}{cccc}
\hline \hline \multicolumn{3}{c}{ Generation $(\mathrm{kWh})$} \\
\hline time & Generator 1 & Generator 2 & Generator 3 \\
\hline 1 & 11.2261 & 8 & 10 \\
2 & 16.6547 & 0 & 10 \\
3 & 14.0833 & 0 & 10 \\
4 & 13.0547 & 0 & 10 \\
5 & 14.2975 & 0 & 10 \\
6 & 13.3119 & 0 & 10 \\
7 & 19.6547 & 0 & 10 \\
8 & 18.05884 & 2.95306 & 10
\end{tabular}




\begin{tabular}{cccc}
9 & 10.73919 & 17.55831 & 10 \\
10 & 10.59923 & 28.38887 & 10 \\
11 & 30 & 46.67285 & 28.37715 \\
12 & 10.41414 & 35.29536 & 10 \\
13 & 10.5542 & 30.1483 & 10 \\
14 & 10.12563 & 20.16717 & 10 \\
15 & 21.00036 & 6.94964 & 10 \\
16 & 28.4881 & 0 & 10 \\
17 & 30 & 0.71197 & 23.46413 \\
18 & 30 & 3.1309 & 10 \\
19 & 30 & 0 & 19.4405 \\
20 & 30 & 6.6239 & 10 \\
21 & 30 & 6.5 & 10 \\
22 & 30 & 3.0333 & 10 \\
23 & 28.4047 & 0 & 10 \\
24 & 24.0333 & 0 & 10 \\
\hline \hline
\end{tabular}
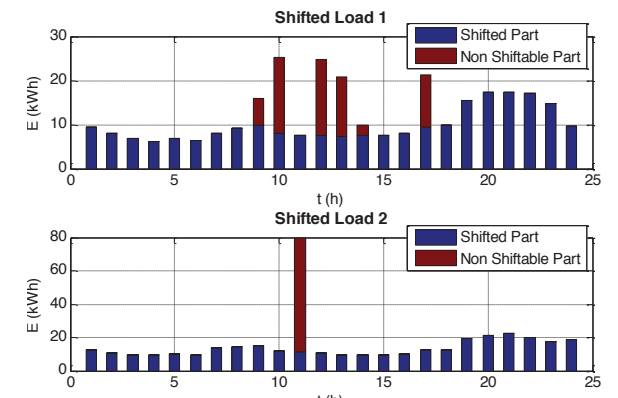

$\stackrel{\text { th }}{\text { Load } 3}$

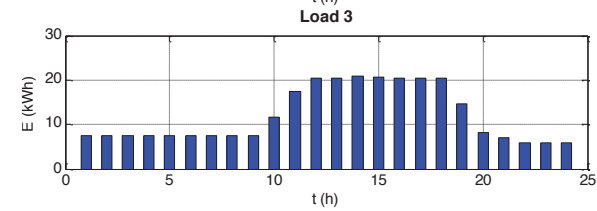

Fig. 8 Scheduling of the loads
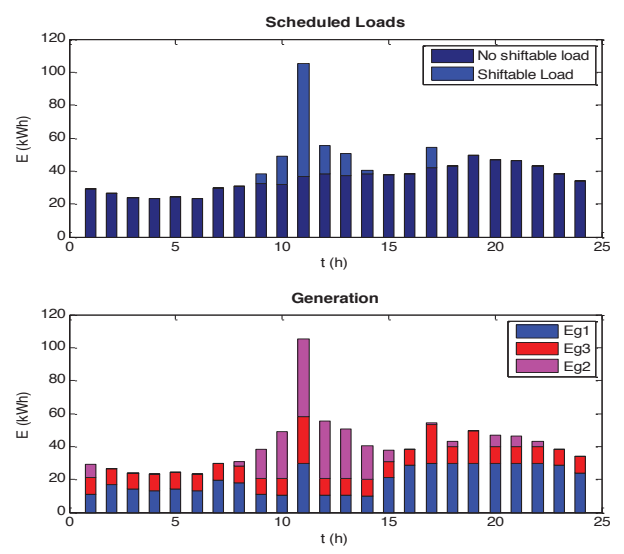

Fig. 9 Demand and generation comparison

\section{CONCLUSION}

In this paper a MILP based EMS has been described and implemented. The idea is to provide a complete two levels architecture for higher level control of islanded micro-grids and the EMS here proposed is one of them. The EMS indeed provides the operating set points to the OPF that finely adjusts the latter to keep frequency within required boundaries and voltage drops below given threshold. In this way, secondary regulation can be skipped and primary regulation can reduce its range of action, limiting the size of the storage of the generation units that have to provide power service to the micro-grid. Further work will be addressed towards the definition of the complete architecture and the execution of laboratory tests on it.

\section{REFERENCES}

[1] J. M. Guerrero et al. "Hierarchical Control of Droop-Controlled AC and DC Microgrids-A General Approach Toward Standardization”, IEEE Trans on Industrial electronics vol 58 issue 1, pp158-172.

[2] Y Riffonneau, S. Bacha, F. Barruel, and S. Ploix, "Optimal powerflow management for grid connected pv systems with batteries," IEEETransactions on Sustainable Energy, vol. 2, no. 3, pp. 309 -320, july2011

[3] Y Hayashi,H . Miyamoto,J . Matsuki,T . lizuka,and H. Azuma," On line optimization method for operation of generators in a micro grid," lEE\}Trans. PE, vol. 128, no. 2, pp. 388-396, 2008.

[4] Dynamic Optimal Schedule Management Method for Microgrid System Considering Forecast Errors of Renewable Power Generations (Sendai, Japan)

[5] E. Riva Sanseverino, N. Nguyen Quang, G. Zizzo, M. L. Di Silvestre, A Madonia, C. Li, J.M. Guerrero, (2013). Optimal Power flow based on glow worm-swarm optimization for three-phase islanded microgrids. In AEIT Annual Conference 2013: Innovation and Scientific and Technical Culture for Development, AEIT 2013 - Selected Proceedings Papers. IEEE Press

[6] N. Nguyen Quang, E. Riva Sanseverino, M. L. Di Silvestre, J. Guerrero, (2013). A parametric study on unbalanced three phase islanded microgrids with inverter interfaced units. In AEIT Annual Conference 2014: Innovation and Scientific and Technical Culture for Development, AEIT 2013 - Selected Proceedings Papers. IEEE Press

[7] J. Rocabert, Gustavo M.S. Azevedo, I. Candela, F. Blaabjerg, P. Rodriguez (2011) Connection and Disconnection Transients for MicroGrids under Unbalance Load Condition. Proceedings of the 14th European Conference on Power Electronics and Applications (EPE 2011),

[8] Rocabert, J. ; Azevedo, G. ; Candela, I. ; Teoderescu, R. ; Rodriguez, P. ; Etxebarria-Otadui, I. (2010) Microgrid connection management based on an intelligent connection agent. - 36th Annual Conference on IEEE Industrial Electronics Society IECON 2010. pp: 3028 - 3033

[9] A.Vaccaro, V. Loia, G.Formato, P.Wall, V.Terzija, (2014). A Self Organizing Architecture for Decentralized Smart Microgrids Synchronization, Control and Monitoring. (2014) Industrial Informatics, IEEE Transactions on (Volume:PP, Issue: 99 ).pp.1-10

[10] E. Mojica-Nava, C. A. Macana, N. Quijano (2014). Dynamic Population Games for Optimal Dispatch on Hierarchical Microgrid Control. IEEE Transactions on Systems, Man, and Cybernetics: Systems. vol. 44, 44, no. 3, march 2014.pp:306-317

[11] IEEE Std. 1547.4-2011 Guide for Design, Operation, and Integration of Distributed Resource Island Systems with Electric Power Systems

[12] Philip P. Barker, Robert W. deMello, "Determining the Impact of Distributed Generation on Power Systems: Part 1 - Radial Distribution Systems", 0-7803-6420-1/00/\$10.(0c)0 2000 IEEE

[13] I. El-Samahy, Ehab El-Saadany, "The Effect of DG on Power Quality in a Deregulated Environment", 0-7803-9156-X/05/\$20.00@2005 IEEE 
[14] W. Li and J. Géza, "Comparison of energy storage system technologies and configurations in a wind farm," in Proc. Power Electronics Specialists Conf. (PESC 2007), Jun. 2007, pp. 1280-1285

[15] M. E. Baran, S. Teleke, L. Anderson, A. Q. Huang, S. Bhattacharya, S. Atcitty, "STATCOM with energy storage for smoothing intermittent wind farm power," in Proc. Power and Energy Soc. General Meeting Conv. and Delivery of Elect. Energy in the 21st Century, Jul. 2008, pp.1-6.

[16] C. Abbey, K. Strunz, and G. Joós, “A knowledge-based approach for control of two-level energy storage for wind energy systems," IEEE Trans. Energy Convers., vol. 24, no. 2, pp. 539-547, Jun. 2009.

[17] G. Koshimizu, T. Nanahara, K. Yoshimoto,H. Hasuike, and T. Shibata, "Subaru project: Application of energy storage for stabilizaion of wind power in power systems," in Proc. EAS 2005 Annu. Meeting Conf. Energy Storage Assoc., Toronto, Canada, 2005.

[18] K. Yoshimoto, T. Nanahara, and G. Koshimizu, "New control method for regulating state-of-charge of a battery in hybrid wind power/battery energy storage system," in Proc. IEEE Power Syst. Conf. and Exposition, 2006, pp. 1244-1251.

[19] X. Li, S. Yu-Jin, and H. Soo-Bin, "Study on power quality control inmultiple renewable energy hybrid micro grid system," in Proc. IEEE Power Tech 2007, Jul. 2007, pp. 2000-2005.

[20] X. Li, Y.-J. Song, and S.-B. Han, "Frequency control in micro-grid power system combined with electrolyzer system and fuzzy PI controller,” J. Power Sources, vol. 180, no. 1, pp. 468-475, May 2008.

[21] R.I.M.Yasser Abdel and S.EhabF.El“"Adaptive Decentralized Droop Controller to Preserve Power Sharing Stability of Paralleled Inverters in Distributed Generation Microgrids”, 0885-8993/\$25.00 (C) 2008 IEEE

[22] RoberH.Lasseter and Paolo Paigi, "Microgrid: Aconceptual Solution", 0-7803-8399-0/04/\$20.a0200 04 IEEE.

[23] F. Katiraei and M. R. Iravani, "Power Management Strategies for a Microgrid With Multiple Distributed Generation Units", IEEE Transactions on Power system, Vol. 21, no. 4, November, 2006

[24] Don O.Koval and Ali A. Chowdhury "Base Load Generator Unit Operating Characteristics”, 0-7803-1877-3/94/\$3.000 1994 IEEE

[25] Don O. Koval and Ali A. Chowdhury "Generating Peaking Unit Operating Characteristics", IEEE Transactions on Industry Applications, vol. 30, no. 5, September/October 1994 